\title{
Restricted-Open-Shell G4(MP2)-Type Procedures
}

\author{
Bun Chan, ${ }^{1}$ Amir Karton, ${ }^{2}$ Krishnan Ragavachari, ${ }^{3}$ Leo Radom ${ }^{4}$
}

${ }^{1}$ Graduate School of Engineering, Nagasaki University, Bunkyo 1-14, Nagasaki 8528521, Japan

${ }^{2}$ School of Chemistry and Biochemistry, The University of Western Australia, Perth, WA 6009, Australia

${ }^{3}$ Department of Chemistry, Indiana University, Bloomington, Indiana 47405, United States

${ }^{4}$ School of Chemistry, University of Sydney, Sydney, NSW 2006, Australia

\section{ABSTRACT}

In the present study, we have reformulated the G4(MP2) and G4(MP2)-6X procedures for use with a restricted-open-shell (RO) formalism. We find that the resulting ROG4(MP2) and ROG4(MP2)-6X procedures generally perform comparably to the original unrestricted (U) variants, including their performance on radicals. Our analysis suggest that this is due mainly to the inclusion of empirical parameters that overcome the slightly less good performance of the $U$ variants. However, a major practical advantage of ROG4(MP2) and ROG4(MP2)-6X is that they can be used in a wider range of computational chemistry software packages than the $U$ analogs. We have demonstrated the importance of this aspect with a large-scale ROG4(MP2)-6X computation for the dissociation of the dodecahedryl dimer $\left(\mathrm{C}_{20} \mathrm{H}_{19}\right)_{2}$. 


\section{INTRODUCTION}

The calculation of thermochemical properties with quantum chemistry procedures represents an important application of computational quantum chemistry. In this regard, composite protocols ${ }^{1}$ such as the $G n$-type methods ${ }^{2}$ have enabled the routine computation of the thermochemistry of medium-sized molecules (up to 30 atoms for $\mathrm{G} n(\mathrm{MP} 2)$-type procedures $\left.{ }^{3,4}\right)$ with high accuracy $\left(\sim 5 \mathrm{~kJ} \mathrm{~mol}^{-1}\right)$. For example, the G4(MP2)-6X procedure ${ }^{4}$ has been applied to diadamantane, anthracene and benzophenone. By exploiting symmetry in quantum chemistry computations, G4(MP2) has been employed for the calculation of $\mathrm{C}_{60 .}{ }^{5}$

We note that Gn-type procedures employ the unrestricted (U) formalism for open-shell systems. This choice (as opposed to restricted-open-shell, RO) may lead to less accurate results when there is significant spin contamination in the reference Hartree-Fock wavefunction. To this end, the Gn-RAD-type procedures ${ }^{6}$ were developed. They use RO reference wavefunctions in order to reduce the impact of excessive spin contamination on the performance. The Gn-RAD-type procedures have been shown to provide better descriptions for systems with moderately-high spin contamination when compared with the Gn-type methods. ${ }^{6}$ In a different manner, the G4-type protocols ${ }^{3,4,7}$ also include a feature in their "higher-level correction" component that is designed to treat open-shell systems comparably well to their treatment of closed-shell species. Such a feature employs distinct parameters for the two types of systems.

As an extension to these previous developments, in the present study, we investigate the use of RO reference wavefunctions for two G4(MP2)-type procedures. This enables us to examine the possibility of providing further improvement for the treatment of open-shell systems. In addition, many commonlyused computational chemistry software packages use the RO formalism exclusively for some quantum chemistry methods that are relevant to G4(MP2)-type protocols. 
With the formulation of RO variants of G4(MP2)-type procedures, we wish to facilitate the use of these methods by reducing software limitations.

\section{COMPUTATIONAL DETAILS}

Standard ab initio molecular orbital theory and density functional theory (DFT) calculations were carried out with the Gaussian $09^{8}$ and Molpro $2012^{9}$ programs. Unless otherwise noted, geometries, zero-point vibrational energies, thermal corrections for $298 \mathrm{~K}$ enthalpies, and benchmark total energies that are used in the present investigation were taken from previous studies.4,10,11 For systems for which the structures were obtained in the present study, the BMK/6-31+G(2df,p) procedure ${ }^{12}$ was employed for geometry optimization and vibrational frequency calculations. Scale factors of 0.9770 and 0.9627 were employed in the calculation of ZPVEs and thermal corrections to enthalpies, respectively, from the BMK vibrational frequencies. ${ }^{4}$ In the present study, the parameters for G4(MP2)-type procedures were fitted to the E2 set. Following previous practice, ${ }^{4,13,14}$ optimization of adjustable parameters involved minimizing the average of the mean absolute deviation (MAD) from benchmark values and the standard deviation (SD) of these deviations. All relative energies are reported in $\mathrm{kJ} \mathrm{mol}^{-1}$.

\section{RESULTS AND DISCUSSION}

Performance of G4(MP2), G4(MP2)-6X, and Their RO Variants for the E2 Set of Thermochemical Properties. We begin our discussion with a brief overview of the performance of the four G4(MP2)-type procedures, namely G4(MP2), G4(MP2)-6X, ROG4(MP2) and ROG4(MP2)-6X, for the E2 set of diverse thermochemical properties. ${ }^{4}$ The E2 set contains fundamental chemical properties such as heats of formation (W4/08, ${ }^{15} \mathrm{G} 2 /$ 97 $^{\prime} \Delta_{\mathrm{f}} \mathrm{H}$ and G3/99' $\Delta_{\mathrm{f}} \mathrm{H}$ ), ionization energies (G2/97 IE), electron affinities (G2/97 EA) and proton affinities (G2/97 PA).16,17 It also covers reaction energies for radical additions (ADD) and radical abstractions (ABS), ${ }^{18}$ barriers for a diverse set of fundamental reactions (DBH24), ${ }^{19}$ and pericyclic reactions (PR8). ${ }^{20}$ In addition, data for hydrogen bonding (HB16) and weak interactions (WI9/04) are also included.21,22 
Table 1. Mean Absolute Deviations (kJ mol-1) from Benchmark Values for the E2 Set and its Subsets for the Various G4(MP2)-type Procedures ${ }^{\mathrm{a}}$

\begin{tabular}{|c|c|c|c|c|}
\hline & G4(MP2) & G4(MP2)-6X & ROG4(MP2) & ROG4(MP2)-6X \\
\hline & \multicolumn{4}{|c|}{ Heats of formation } \\
\hline W4/08 & 4.8 & 4.0 & 4.7 & 4.4 \\
\hline $\mathrm{G} 2 / 97^{\prime} \Delta_{\mathrm{f}} \mathrm{H}$ & 3.8 & 3.0 & 3.6 & 3.2 \\
\hline \multirow[t]{2}{*}{$\mathrm{G} 3 / 99^{\prime} \Delta_{\mathrm{f}} \mathrm{H}$} & 4.5 & 3.4 & 4.5 & 3.4 \\
\hline & \multicolumn{4}{|c|}{ Other fundamental properties } \\
\hline G2/97 IE & 5.0 & 4.7 & 4.9 & 4.6 \\
\hline G2/97 EA & 5.4 & 5.3 & 5.2 & 5.1 \\
\hline \multirow[t]{2}{*}{ G2/97 PA } & 2.4 & 2.8 & 2.4 & 2.6 \\
\hline & \multicolumn{4}{|c|}{ Reaction energies } \\
\hline ADD & 2.4 & 2.5 & 2.6 & 2.7 \\
\hline \multirow[t]{2}{*}{ ABS } & 2.0 & 1.7 & 1.8 & 1.4 \\
\hline & \multicolumn{4}{|c|}{ Reaction barriers } \\
\hline DBH24 & 2.6 & 3.0 & 3.1 & 3.3 \\
\hline \multirow[t]{2}{*}{ PR8 } & 4.3 & 4.4 & 4.3 & 3.6 \\
\hline & \multicolumn{4}{|c|}{ Non-covalent interactions } \\
\hline HB16 & 1.8 & 1.8 & 1.8 & 2.0 \\
\hline \multirow[t]{2}{*}{ WI9/04 } & 3.3 & 1.3 & 3.3 & 2.0 \\
\hline & \multicolumn{4}{|c|}{ Overall } \\
\hline E2 & 4.2 & 3.6 & 4.2 & 3.7 \\
\hline
\end{tabular}

We note that the parameters in the higher-level correction (HLC) of G4(MP2)-6X have been optimized for the E2 set, whereas those in G4(MP2) were determined 
with the G3/05 set. ${ }^{23}$ To enable a more equitable comparison among the four protocols, we use a slightly revised G4(MP2) for which the HLC parameters have been reoptimized for the E2 set. The mean absolute deviations (MADs) from benchmark values for the E2 set and its subsets for the four G4(MP2)-type methods are shown in Table 1.

For the complete E2 set, the MAD for G4(MP2) is $4.2 \mathrm{~kJ} \mathrm{~mol}^{-1}$. The inclusion of the six additional parameters in G4(MP2)-6X leads to a smaller overall MAD of $3.6 \mathrm{~kJ}$ $\mathrm{mol}^{-1}$. This observation is completely consistent with the results of ref 4 . We note that major improvements can be seen for heats of formation, i.e., the W4/08, G2/97' $\Delta_{\mathrm{f}} \mathrm{H}$ and G3/99' $\Delta_{\mathrm{f}} \mathrm{H}$ sets, as well as for the WI9/04 set of weak interactions.

A comparison of G4(MP2) with its R0 analog, i.e., ROG4(MP2), shows that there is very little difference in performance between the two methods, not only for the overall performance, but also for all the subsets of E2. Likewise, there is also little difference between G4(MP2)-6X and ROG4(MP2)-6X for E2 and its subsets. We note that the performance of the previously developed G3-RAD(5d) variant of the G3RAD procedure, in which the only difference compared with G3 is the use of RO reference wavefunctions in G3-RAD(5d) versus $\mathrm{U}$ for $\mathrm{G} 3$, is also quite similar to that for G3. ${ }^{6}$

What might be the reason behind the similarity in performance shown by the $\mathrm{U}$ and RO variants? Do the $U$ and RO reference wavefunctions not lead to a difference in the relative energies, or are the empirical parameters able to compensate for the differences in the underlying quantum chemistry methods? In order to gain some insight into this aspect, we have examined the performance of modified G4(MP2) and ROG4(MP2) procedures (no par) in which the HLC terms are not included. The overall MADs for the E2 set for these two methods are 38.4 for G4(MP2)(no par) and 35.5 for ROG4(MP2)(no par) $\mathrm{kJ} \mathrm{mol}^{-1}$. Thus, it seems that there are small differences between the underlying electronic energies for the two procedures, with RO performing slightly better. 
Table 2. Empirical Parameters Optimized with the E2 Set for G4(MP2)-Type Procedures $^{\mathbf{a}}$

\begin{tabular}{|c|c|c|c|c|}
\hline & G4(MP2) & G4(MP2)-6X & ROG4(MP2) & ROG4(MP2)-6X \\
\hline A & 9.5363 & 7.1726 & 9.2889 & 7.1240 \\
\hline $\mathrm{A}^{\prime}$ & 9.6735 & 7.2642 & 9.4476 & 7.2585 \\
\hline B & 4.1858 & 3.6767 & 3.7077 & 3.2301 \\
\hline $\mathrm{C}$ & 9.7966 & 7.2387 & 9.8100 & 7.2555 \\
\hline $\mathrm{D}$ & 2.1499 & 2.4045 & 2.1299 & 2.2866 \\
\hline $\mathrm{E}$ & 2.2595 & 1.0215 & 2.4113 & 1.2669 \\
\hline $\mathrm{c}[\mathrm{MP} 20 \mathrm{~S} / \mathrm{sm}]$ & 1.0000 & 1.3269 & 1.0000 & 1.2369 \\
\hline $\mathrm{c}[\mathrm{MP} 2 \mathrm{SS} / \mathrm{sm}]$ & 1.0000 & 0.4032 & 1.0000 & 0.4840 \\
\hline c[MP2OS/lg] & 1.0000 & 1.2488 & 1.0000 & 1.2638 \\
\hline c[MP2SS/lg] & 1.0000 & 0.4855 & 1.0000 & 0.5396 \\
\hline $\mathrm{c}[\Delta \mathrm{CCSD}]$ & 1.0000 & 1.0773 & 1.0000 & 0.9792 \\
\hline$c[\Delta(\mathrm{T})]$ & 1.0000 & 0.8240 & 1.0000 & 0.8473 \\
\hline
\end{tabular}

This observation, together with the similarity in the performance for G4(MP2) and ROG4(MP2), suggests that differences in the HLC parameters compensate for the slightly worse underlying performance for the unrestricted G4(MP2) protocol. The E2-optimized HLC and scaling parameters for G4(MP2), G4(MP2)-6X, ROG4(MP2) and ROG4(MP2)-6X are shown in Table 2. We can see that there is a notable difference between the optimized values for parameter B of G4(MP2) and ROG4(MP2). Likewise, the value of B for ROG4(MP2)-6X is quite different from that for G4(MP2)-6X. In addition, parameters E, c[MP2SS/sm] and c[MP2SS/lg] for ROG4(MP2)-6X also have fairly different optimized values when compared with those for G4(MP2)-6X. Thus, the empirical parameters in the G4(MP2)-type procedures indeed appear to be compensating for some of the shortcoming of the 
underlying quantum chemistry method, to the extent that the use of $\mathrm{U}$ and RO reference wavefunctions do not result in a significant difference in the overall performance when a wide range of systems is considered.

Performance for Radicals. We have seen in the previous section that the overall performances of G4(MP2)-type procedures are not substantially affected by the change in the underlying reference wavefunctions from $U$ to $R O$ when the effect of reoptimizing the parameters is taken into account. However, in cases where radicals are involved, there may be more significant differences between the $U$ and RO variants. Thus, we have examined the performance of the G4(MP2)-type procedures for the open-shell doublet radicals in the W4/08 set. The $S^{2}$ values for these species and the deviations from benchmark values for G4(MP2), G4(MP2)-6X, and their RO variants are shown in Table 3, together with the associated statistical measures.

Table 3. Open-Shell Doublet Radicals in the W4/08 Set, Their $S^{2}$ Values, Deviations ( $\mathrm{kJ} \mathrm{mol}^{-1}$ ) from Benchmark Atomization Energies for the G4(MP2), G4(MP2)-6X, ROG4(MP2) and ROG4(MP2)-6X Procedures, and Overall

\section{Statistical Performances ${ }^{a}$}

$\begin{array}{lrrrcc} & \mathrm{S}^{2} & \text { G4(MP2) } & \text { G4(MP2)-6X } & \text { ROG4(MP2) } & \text { ROG4(MP2)-6X } \\ \mathrm{H}_{2} \mathrm{CN} & 0.761 & 2.1 & 2.1 & 1.6 & 1.7 \\ \mathrm{CH}_{2} \mathrm{NH}_{2} & 0.754 & -3.3 & -3.4 & -5.1 & -3.7 \\ \mathrm{CH}_{3} \mathrm{NH} & 0.754 & -1.2 & -1.5 & -3.1 & -1.3 \\ \mathrm{~N}_{2} \mathrm{H} & 0.756 & -1.7 & -1.0 & -2.1 & -0.8 \\ \mathrm{FO}_{2} & 0.770 & -9.8 & -6.3 & -11.0 & -11.9 \\ \mathrm{OClO} & 0.756 & -5.5 & -1.4 & -1.7 & 0.7 \\ \mathrm{ClOO} & 0.770 & -22.2 & -19.5 & -13.6 & -15.1 \\ \mathrm{OH} & 0.752 & 2.3 & 2.0 & 0.3 & 1.0 \\ \mathrm{CH} & 0.753 & 7.4 & 5.4 & 6.4 & 5.7\end{array}$




$\begin{array}{lrrrrr}\mathrm{CH}_{3} & 0.754 & 2.0 & 1.3 & 2.1 & 2.5 \\ \mathrm{C}_{2} \mathrm{H} & 0.780 & -3.0 & -4.5 & 0.1 & -2.9 \\ \mathrm{CN} & 0.766 & -0.4 & -0.1 & 3.1 & 1.8 \\ \mathrm{NO} & 0.753 & 2.7 & 4.0 & 1.5 & 3.3 \\ \mathrm{OF} & 0.753 & -2.0 & 0.1 & -3.3 & -2.0 \\ \mathrm{HS} & 0.753 & 2.1 & -1.1 & -0.2 & -1.7 \\ \mathrm{ClO} & 0.755 & -6.0 & -5.6 & -10.5 & -10.6 \\ \mathrm{NH}_{2} & 0.753 & 1.0 & 0.0 & -0.9 & 0.0 \\ \mathrm{SiH} & 0.754 & 4.8 & 3.2 & 3.6 & 3.7 \\ \mathrm{SiF} & 0.754 & 4.9 & 3.9 & 4.2 & 4.2 \\ \mathrm{CF}^{2} & 0.753 & 6.5 & 5.4 & 6.5 & 5.7 \\ \mathrm{CH}_{2} \mathrm{CH} & 0.763 & 3.6 & 3.0 & 3.8 & 2.0 \\ \mathrm{HCO}^{2} & 0.754 & 4.0 & 4.5 & 3.0 & 3.5 \\ \mathrm{NO}_{2} & 0.755 & 1.3 & 4.0 & 1.0 & 3.4 \\ \mathrm{HO}_{2} & 0.754 & -2.3 & 0.5 & -4.2 & -3.2 \\ \mathrm{~S}_{2} \mathrm{H} & 0.754 & 2.7 & -3.6 & -0.6 & -5.6 \\ \mathrm{MAD}^{\mathrm{b}} & & 4.2 & 3.5 & 3.7 & 3.9 \\ \mathrm{MD}^{\mathrm{c}} & & -0.4 & -0.4 & -0.8 & -0.8 \\ \mathrm{SD}^{\mathrm{d}} & & 6.1 & 5.2 & 5.1 & 5.4 \\ \mathrm{LD}^{\mathrm{e}} & & -22.2 & -19.5 & -13.6 & -15.1\end{array}$

a $S^{2}$ values obtained from UHF calculations using the modified AVQZ basis set defined in G4(MP2). b Mean absolute deviation. c Mean deviation. d Standard deviation among the deviations. ${ }^{\mathrm{e}}$ Largest deviation.

It can be seen that, while there are substantial variations in some individual cases, the overall statistics for the four G4(MP2)-type methods, do not vary to a large extent. Although the extent of spin contamination in these systems is not very large 
$\left(\left\langle\mathrm{S}^{2}\right\rangle \leq 0.78\right)$, the more spin-contaminated systems do appear to lead to some of the larger differences between the $\mathrm{U}$ and RO results. However, there is not always an improvement in going from $U$ to $R O$. When no empirical parameters are incorporated, the G4(MP2)(no par) and ROG4(MP2)(no par) methods lead to MADs of 32.9 and $29.1 \mathrm{~kJ} \mathrm{~mol}^{-1}$, respectively.

We note that the species in Table 3 have fairly mild degrees of spin contamination. We have thus examined an additional set of five species with larger $\left\langle\mathrm{S}^{2}\right\rangle$ values (Table 4). ${ }^{24}$ We have obtained atomization energies using the higherlevel W1X-1 ${ }^{25}$ and $\mathrm{W} 2 \mathrm{X}^{26}$ procedures for these systems (see Supporting Information), with the W2X atomization energies, being our highest-level values, used as our benchmark values. It can be seen that, in a similar manner to the W4/08 set of doublet radicals, ROG4(MP2)(no par) somewhat outperforms G4(MP2)(no par) for these species. However, when empirical HLC parameters and scaling coefficients are included, ROG4(MP2) and ROG4(MP2)-6X do not seem to be more accurate than the $U$ variants for these five species.

Thus, the use of an RO reference wavefunction leads to a small improvement in the overall performance of the underlying quantum chemistry component when compared with that for the U method. However, the inclusion of the HLC and scaling parameters essentially eliminates the advantage of using RO wavefunctions in an average sense for this set of open-shell molecules. To this end, we note that our observations are consistent with the findings in ref 6 . Thus, for the heats of formation of radical species in the G2/97 set, it was shown that the G3 and G2RAD(5d) procedures yield very similar MADs. 
Table 4. Open-Shell Doublet Radicals with Relatively Large $\mathrm{S}^{2}$ Values and Deviations (kJ mol-1) from Benchmark W2X Atomization Energies for the G4(MP2), G4(MP2)-6X, ROG4(MP2), ROG4(MP2)-6X G4(MP2)(no par) and ROG4(MP2)(no par) Procedures

\begin{tabular}{lccccccc} 
& $\mathrm{S}^{2}$ & G4(MP2) & $\begin{array}{c}\text { G4(MP2) } \\
-6 \mathrm{X}\end{array}$ & $\begin{array}{c}\text { ROG4(MP2) } \\
\text { ROG4(MP2) }\end{array}$ & $\begin{array}{c}\text { G4(MP2) } \\
\text { (no par) }\end{array}$ & $\begin{array}{c}\text { ROG4(MP2) } \\
\text { (no par) }\end{array}$ \\
$\mathrm{CH}_{2} \mathrm{CCH}$ & 0.967 & 0.9 & 0.6 & 0.7 & 0.0 & -13.6 & -12.6 \\
$\mathrm{CH}_{2} \mathrm{CHCH}_{2}$ & 0.953 & 0.2 & -0.1 & 2.0 & 1.6 & -17.7 & -14.6 \\
$\mathrm{CH}_{2} \mathrm{CHO}$ & 0.922 & 0.6 & 0.9 & -0.1 & 0.0 & -13.9 & -13.2 \\
$\mathrm{CH}_{2} \mathrm{CN}$ & 0.951 & 0.6 & 0.5 & 0.4 & 0.3 & -13.9 & -12.9 \\
$\mathrm{CH}_{2} \mathrm{Ph}$ & 1.345 & -1.2 & -2.1 & 4.6 & 3.0 & -35.7 & -27.4 \\
\hline
\end{tabular}


Application to Larger System. As noted earlier, the use of RO reference wavefunctions for the ROG4(MP2) and ROG4(MP2)-6X procedures also opens up the possibility of using a wider range of computational chemistry software packages for the calculations with these methods. As an example, we have employed the Molpro program suite to compute the $298 \mathrm{~K}$ bond dissociation enthalpy (BDE) of the $\mathrm{C}_{20} \mathrm{H}_{19}-\mathrm{C}_{20} \mathrm{H}_{19}$ dimer of dodecahedryl radical (Figure 1), using ROG4(MP2)-6X. To the best of our knowledge, this represents one of the largest quantum chemistry computations carried out using a G4(MP2)-type procedure.

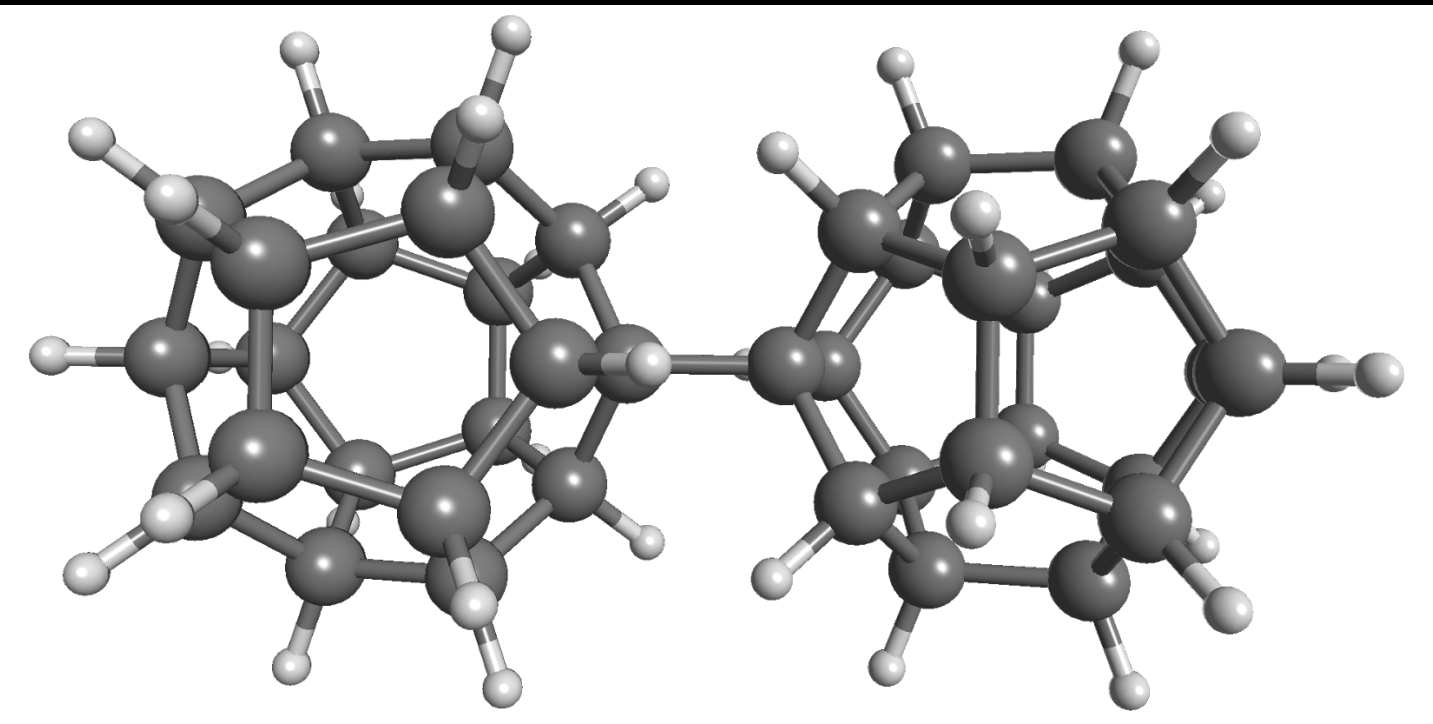

Figure 1. Dodecahedryl dimer $\left(\mathrm{C}_{20} \mathrm{H}_{19}-\mathrm{C}_{20} \mathrm{H}_{19}\right)$ with a computed ROG4(MP2)-6X bond dissociation enthalpy of $372.6 \mathrm{~kJ} \mathrm{~mol}^{-1}$.

We obtain a value of $372.6 \mathrm{~kJ} \mathrm{~mol}^{-1}$ for this quantity (Table 5). In comparison, we have also computed the BDE using a variety of DFT procedures and their dispersioncorrected variants. These include: B3-LYP, ${ }^{27}$ BH\&H-LYP, ${ }^{28}$ CAM-B3-LYP, ${ }^{29}$ PBE0, ${ }^{30}$ TPSSh, ${ }^{31}$ LC- $\omega$ PBE, ${ }^{32}$ B1-B95, 33 MPW1-B95, ${ }^{34}$ and MPW-B1K. ${ }^{34}$ It can be seen that the three methods based on the B95 correlation functional, namely B1-B95, MPW1B95 and MPW-B1K yield good agreement with ROG4(MP2)-6X when a dispersion correction term is included. It is noteworthy that dispersion corrections ${ }^{35,36,37}$ make sizable contributions of $\sim 35-70 \mathrm{~kJ} \mathrm{~mol}^{-1}$ to the DFT-D3BJ BDEs. This is somewhat 
intriguing as the BDE of $372.6 \mathrm{~kJ} \mathrm{~mol}^{-1}$ is not too different from that of a typical C-C bond in small alkanes, e.g., 377.9 and $365.6 \mathrm{~kJ} \mathrm{~mol}^{-1}$, respectively, for $\mathrm{CH}_{3}-\mathrm{CH}_{3}$ and $\left(\mathrm{CH}_{3}\right)_{3} \mathrm{C}-\mathrm{CH}_{3}$, for which the contribution of dispersion to the BDEs would be expected to be quite small. Thus, it seems that the large dispersion attraction between the two $\mathrm{C}_{20} \mathrm{H}_{19}$ fragments in $\mathrm{C}_{20} \mathrm{H}_{19}-\mathrm{C}_{20} \mathrm{H}_{19}$ is opposed by a repulsive force of similar magnitude, leading to a $\mathrm{C}-\mathrm{C}$ BDE that is not too different from those of much smaller hydrocarbons.

Table 5. Bond Dissociation Enthalpies $\left(298 \mathrm{~K}^{\mathrm{kJ} \mathrm{mol}}{ }^{-1}\right)$ for the $\mathrm{C}-\mathrm{C}$ Bond Between the Two Dodecahedryl $\left(\mathrm{C}_{20} \mathrm{H}_{19}\right)$ Fragments in $\mathrm{C}_{20} \mathrm{H}_{19}-\mathrm{C}_{20} \mathrm{H}_{19}$ Calculated with a Variety of DFT Procedures and Their Dispersion-Corrected (D3BJ) Variants, and the D3BJ Contributions [E(D3BJ)] to the BDEs.

\begin{tabular}{|c|c|c|c|}
\hline & DFT & DFT-D3BJ & $\mathrm{E}(\mathrm{D} 3 \mathrm{BJ})$ \\
\hline B3-LYP & 275.6 & 346.7 & 71.0 \\
\hline BH\&H-LYP & 288.4 & 343.9 & 55.6 \\
\hline CAM-B3-LYP & 306.2 & 353.1 & 46.9 \\
\hline PBE0 & 308.0 & 354.3 & 46.3 \\
\hline TPSSh & 276.5 & 335.7 & 59.3 \\
\hline LC- $\omega$ PBE & 322.9 & 373.9 & 51.0 \\
\hline B1-B95 & 318.0 & 373.5 & 55.5 \\
\hline MPW1-B95 & 330.7 & 366.3 & 35.6 \\
\hline MPW-B1K & 338.4 & 373.5 & 35.1 \\
\hline ROG4(MP2)-6X & & & \\
\hline
\end{tabular}

\section{CONCLUDING REMARKS}

In the present study, we have reformulated the G4(MP2) and G4(MP2)-6X procedures to be used with a restricted-open-shell (RO) formalism. The resulting ROG4(MP2) and ROG4(MP2)-6X procedures have been assessed with the E2 set of 
diverse thermochemical properties derived from a mixture of both closed- and open-shell species. We find that the average performance of the RO variants is similar to that for the original G4(MP2)-type procedures. Our analysis suggests that this is due mainly to the inclusion of the empirical parameters that are optimized for each procedure, which compensates for the slightly less good performance of the $U$ variants. A major practical advantage of ROG4(MP2) and ROG4(MP2)-6X is that they can be used in a wider range of computational chemistry software packages than the $\mathrm{U}$ analogs. We have demonstrated this aspect by conducting a large-scale ROG4(MP2)-6X computation using the dissociation of the dodecahedryl dimer $\left(\mathrm{C}_{20} \mathrm{H}_{19}\right)_{2}$ as an example.

\section{ASSOCIATED CONTENT}

Supporting Information. Total electronic energies for Gn(MP2)-type procedures for the E2 set (Table S1), deviations from benchmark values (Table S2), and energies associated with $\mathrm{C}_{20} \mathrm{H}_{19}$ and its dimer (Table S3). This material is available free of charge via the Internet at http://pubs.acs.org

\section{ACKNOWLEDGEMENTS}

We gratefully acknowledge funding from the Australian Research Council (to L.R. and A.K.), National Science Foundation (to K.R.), and Japan Society for the Promotion of Science and Nagasaki University (to B.C.), and generous grants of computer time from the NCI National Facility, Intersect Australia Ltd, RIKEN ACCC and the Institute for Molecular Science.

\section{REFERENCES}

1 Karton, A. WIREs Comput. Mol. Sci. 2016, 6, 292-310.

2 Curtiss, L. A.; Redfern, P. C.; Raghavachari, K. WIREs Comput. Mol. Sci. 2011, 1, 810-825.

3 Curtiss, L. A.; Redfern, P. C.; Raghavachari, K. J. Chem. Phys. 2007, 126, 084108$1-12$.

4 Chan, B.; Deng, J.; Radom, L. J. Chem. Theory Comput. 2011, 7, 112-120. 
5 Wan, W.; Karton, A. Chem. Phys. Lett. 2016, 643, 34-38.

6 Henry, D. J.; Sullivan, M. B.; Radom, L. J. Chem. Phys. 2003, 118, 4849-4860.

7 Curtiss, L. A.; Redfern, P. C.; Raghavachari, K. J. Chem. Phys. 2007, 126, 084108$1-12$.

8 Frisch, M. J.; Trucks, G. W.; Schlegel, H. B.; Scuseria, G. E.; Robb, M. A.; Cheeseman, J. R.; Scalmani, G.; Barone, V.; Mennucci, B.; Petersson, G. A.; Nakatsuji, H.; Caricato, M.; Li, X.; Hratchian, H. P.; Izmaylov, A. F.; Bloino, J.; Zheng, G.; Sonnenberg, J. L.; Hada, M.; Ehara, M.; Toyota, K.; Fukuda, R.; Hasegawa, J.; Ishida, M.; Nakajima, T.; Honda, Y.; Kitao, O.; Nakai, H.; Vreven, T.; Montgomery, Jr., J. A.; Peralta, J. E.; Ogliaro, F.; Bearpark, M.; Heyd, J. J.; Brothers, E.; Kudin, K. N.; Staroverov, V. N.; Kobayashi, R.; Normand, J.; Raghavachari, K.; Rendell, A.; Burant, J. C.; Iyengar, S. S.; Tomasi, J.; Cossi, M.; Rega, N.; Millam, N. J.; Klene, M.; Knox, J. E.; Cross, J. B.; Bakken, V.; Adamo, C.; Jaramillo, J.; Gomperts, R.; Stratmann, R. E.; Yazyev, O.; Austin, A. J.; Cammi, R.; Pomelli, C.; Ochterski, J. W.; Martin, R. L.; Morokuma, K.; Zakrzewski, V. G.; Voth, G. A.; Salvador, P.; Dannenberg, J. J.; Dapprich, S.; Daniels, A. D.; Farkas, Ö.; Foresman, J. B.; Ortiz, J. V.; Cioslowski, J.; Fox, D. J. Gaussian 09, Revision C.01; Gaussian, Inc.: Wallingford CT, 2009.

9 Werner, H.-J.; Knowles, P. J.; Knizia, G.; Manby, F. R.; Schütz, M. WIREs Comput. Mol. Sci. 2012, 2, 242-253.

10 Chan, B.; Radom, L. J. Chem. Theory Comput. 2011, 7, 2852-2863.

11 Chan, B.; Gill, P. M. W.; Radom, L. J. Chem. Theory Comput. 2012, 8, 4899.

12 Boese, A. D.; Martin, J. M. L. J. Chem. Phys. 2004, 121, 3405-3416.

13 Chan, B.; Radom, L. J. Chem. Theory Comput. 2013, 9, 4769-4778.

14 Chan, B.; Radom, L. Theor. Chem. Acc. 2014, 133, 1426-1-9.

15 Karton, A.; Tarnopolsky, A. Lamere, J.-F.; Schatz, G. C.; Martin, J. M. L. J. Phys. Chem. A 2008, 112, 12868-12886.

16 Curtiss, L. A.; Redfern, P. C.; Raghavachari, K.; Pople, J. A. J. Chem. Phys. 1997, 106, 1063-1079. 
17 Curtiss, L. A.; Redfern, P. C.; Raghavachari, K.; Pople, J. A. J. Chem. Phys. 1998, 109, 42-55.

18 Lin, C. Y.; Hodgson, J. L.; Namazian, M.; Coote, M. L. J. Phys. Chem. A 2009, 113, 3690-3697.

19 Zheng, J.; Zhao, Y.; Truhlar, D. G. J. Chem. Theory Comput. 2009, 5, 808-821.

20 Ess, D. H.; Houk, K. N. J. Phys. Chem. A 2005, 109, 9542-9553.

21 Boese, A. D.; Martin, J. M. L.; Klopper, W. J. Phys. Chem. A 2007, 111, 1112211133.

22 Zhao, Y.; Truhlar, D. G. J. Chem. Theory Comput. 2005, 1, 415-432.

23 Curtiss, L. A.; Redfern, P. C.; Raghavachari, K. J. Chem. Phys. 2005, 123, $124107-$ $1-12$.

24 Menon, A. S.; Radom, L. J. Phys. Chem. A 2008, 112, 13225-13230.

25 Chan, B.; Radom, L. J. Chem. Theory Comput. 2012, 8, 4259-4269.

26 Chan, B.; Radom, L. J. Chem. Theory Comput. 2015, 11, 2109-2119.

27 Stephens, P. J.; Devlin, F. J.; Chabalowski, C. F.; Frisch, M. J. J. Phys. Chem. 1994, 98, 11623-11627.

28 Becke, A. D. J. Chem. Phys. 1993, 98, 1372-1377.

29 Yanai, T.; Tew, D.; Handy, N. Chem. Phys. Lett. 2004, 393, 51-57.

30 Adamo, C.; Barone, V. J. Chem. Phys. 1999, 110, 6158-6170.

31 Staroverov, V. N.; Scuseria, G. E.; Tao, J.; Perdew, J. P. J. Chem. Phys. 2003, 119, 12129-12137.

32 Vydrov, O. A.; Scuseria, G. E. J. Chem. Phys. 2006, 125, 34109-1-9.

33 Becke, A. D. J. Chem. Phys. 1996, 104, 1040-1046.

34 Zhao, Y.; Truhlar, D. G. J. Phys. Chem. A 2004, 108, 6908-6918.

35 Becke, A. D.; Johnson, E. R. J. Chem. Phys. 2005, 123, 154101-1-9.

36 Grimme, S.; Ehrlich, S.; Goerigk, L. J. Comput. Chem. 2011, 32, 1456-1465.

37 Grimme, S. WIREs Comput. Mol. Sci. 2011, 1, 211-228. 